\title{
Organizational contextual features that influence the implementation of evidence- based practices across healthcare settings: a systematic integrative review
}

Shelly-Anne Li ${ }^{1,4^{*}}$ (D, Lianne Jeffs ${ }^{2,3}$, Melanie Barwick ${ }^{4,5,6}$ and Bonnie Stevens ${ }^{1,4,7}$

\begin{abstract}
Background: Organizational contextual features have been recognized as important determinants for implementing evidence-based practices across healthcare settings for over a decade. However, implementation scientists have not reached consensus on which features are most important for implementing evidence-based practices. The aims of this review were to identify the most commonly reported organizational contextual features that influence the implementation of evidence-based practices across healthcare settings, and to describe how these features affect implementation.

Methods: An integrative review was undertaken following literature searches in CINAHL, MEDLINE, PsycINFO, EMBASE, Web of Science, and Cochrane databases from January 2005 to June 2017. English language, peer-reviewed empirical studies exploring organizational context in at least one implementation initiative within a healthcare setting were included. Quality appraisal of the included studies was performed using the Mixed Methods Appraisal Tool. Inductive content analysis informed data extraction and reduction.

Results: The search generated 5152 citations. After removing duplicates and applying eligibility criteria, 36 journal articles were included. The majority $(n=20)$ of the study designs were qualitative, 11 were quantitative, and 5 used a mixed methods approach. Six main organizational contextual features (organizational culture; leadership; networks and communication; resources; evaluation, monitoring and feedback; and champions) were most commonly reported to influence implementation outcomes in the selected studies across a wide range of healthcare settings.

Conclusions: We identified six organizational contextual features that appear to be interrelated and work synergistically to influence the implementation of evidence-based practices within an organization. Organizational contextual features did not influence implementation efforts independently from other features. Rather, features were interrelated and often influenced each other in complex, dynamic ways to effect change. These features corresponded to the constructs in the Consolidated Framework for Implementation Research (CFIR), which supports the use of CFIR as a guiding framework for studies that explore the relationship between organizational context and implementation. Organizational culture was most commonly reported to affect implementation. Leadership exerted influence on the five other features, indicating it may be a moderator or mediator that enhances or impedes the implementation of evidence-based practices. Future research should focus on how organizational features interact to influence implementation effectiveness.
\end{abstract}

Keywords: Organizational context, Implementation, Knowledge translation, Evidence-based practice, Healthcare, Adoption, Organization, Context, Integrative review

\footnotetext{
* Correspondence: shellyanne.li@mail.utoronto.ca

${ }^{1}$ Lawrence S. Bloomberg Faculty of Nursing, University of Toronto, Toronto,

ON, Canada

${ }^{4}$ Child Health Evaluative Sciences, Research Institute, Peter Gilgan Centre for Research and Learning, The Hospital for Sick Children, Toronto, ON, Canada Full list of author information is available at the end of the article
}

(c) The Author(s). 2018 Open Access This article is distributed under the terms of the Creative Commons Attribution 4.0 International License (http://creativecommons.org/licenses/by/4.0/), which permits unrestricted use, distribution, and reproduction in any medium, provided you give appropriate credit to the original author(s) and the source, provide a link to the Creative Commons license, and indicate if changes were made. The Creative Commons Public Domain Dedication waiver (http://creativecommons.org/publicdomain/zero/1.0/) applies to the data made available in this article, unless otherwise stated. 


\section{Background}

Each year, at least $\$ 160$ billion is allocated to medical and health research expenditures in North America [1, 2]. Despite major financial investments and advancements in knowledge generation for evidence-based practices (EBPs), healthcare organizations encounter significant implementation failures or challenges [3]. EBP entails making decisions about how to provide or promote healthcare by integrating the best available research evidence with clinical expertise and patient values and preferences [4]. A variety of definitions for the term "implementation" exists in health research. In this review, implementation is defined as "the use of strategies to adopt and integrate evidence-based health interventions and change practice patterns within specific settings" [5]. The estimated average evidence-to-practice time lag is 17 years [6]. This "know-do" gap can result in suboptimal care or a delay in benefits associated with unsuccessful implementations [7]. While provider-level characteristics such as knowledge, attitudes, and behavior about the EBP are widely acknowledged to be critical in addressing this know-do gap, organizational contextual features have also been recognized as a key consideration when implementing EBPs in healthcare settings [7-9]. Over the last decade, addressing this gap has been a priority research focus in implementation science. One such focus has been the need to better understand the role organizational contextual features play in supporting or hindering implementation $[10,11]$.

Currently, there are multiple definitions for the term "organizational context" in various disciplines. Quality improvement (QI) literature appears to establish parameters around this term. Glasgow et al. [12] developed an analytic framework to describe how organizational context modifies QI. The authors described how the intrinsic organizational features such as staffing and culture, facility structure, and QI experience together make up the organizational context of a QI initiative. Extant organizational management literature appears to have the most mature conceptualization of organizational context, often including components such as organizational culture, climate, goals and missions, processes (policies, mode of governance), power dynamics, state/condition, structure (size, shape and type of organization, hierarchical levels), and time [12-14]. Context is commonly depicted in three levels, and researchers tend to reserve the term "organizational context" for internal organizational features. The macro level recognizes the influence of political-economic forces, which focuses on interactions between markets and societies at the broadest level. The meso level represents organizational characteristics such as culture, climate, tacit rules, and shared meanings that influence individual behaviors $[15,16]$. The micro level consists of activities in the local setting that provide a contextual influence. Together, these levels of context form a complex set of influences on organizations [15].

The relationship between implementation outcomes and context have been described in implementation theories, models, and frameworks including Rogers' diffusion of innovations theory [17]; the Consolidated Framework for Implementation Research framework (CFIR) [18]; the Exploration, Preparation, Implementation, and Sustainment framework [19]; the Integrated Promoting Action Research in Health Services framework (i-PARiHS) [20]; and the Theoretical Domains Framework [21]. The implementation theories, models, and frameworks appear to characterize context as a multi-dimensional concept that interacts with different phases of knowledge translation (KT).

\section{Problem identification}

While these implementation frameworks include context, no single framework is sufficiently comprehensive about what comprises context. In addition, the authors of the frameworks are often inconsistent in how context is theoretically and operationally defined. Without a shared understanding of context and its characteristics and features, there is little direction to which features of context are most influential to KT efforts [22]. Extending beyond conceptual theories, models, and frameworks; this review aims to synthesize and summarize organizational contextual features commonly reported to influence the implementation of EBPs in actual healthcare settings.

\section{Methods}

The guiding question for the review was the following: Which organizational contextual features are most commonly reported to influence the implementation of EBP in healthcare settings? Studies with diverse study designs and methods (qualitative, quantitative, mixed methods) that explored, described, or measured organizational contextual features in implementation research were included in this review [23]. Only empirical literature was included. Methodological rigor was informed by Whittemore and Knafl's [24] five-phase integrative review method: problem identification (noted above), literature search, data evaluation, data analysis, and result presentation.

\section{Literature search}

The search strategy (see Additional file 1) was developed on MEDLINE in consultation with two librarians and applied to Cochrane databases, CINAHL, MEDLINE, EMBASE, PsycINFO, and gray literature. The journal Implementation Science (from journal inception to June 2017) was hand searched to uncover additional relevant articles. The search included four categories of search 
key terms: (a) Implementation, (b) Context, (c) Evidence, and (d) Organization. Inclusion and exclusion criteria (Table 1) were applied during screening.

\section{Data evaluation}

Following the removal of duplicates, two reviewers (SAL, PEA) independently double-screened the titles and abstracts of a randomly selected sample (generated from an online randomized website) of $20 \%(n=1034)$ of the retrieved citations to ensure interrater reliability. Once suitable agreement $(k=0.85)$ was achieved, title and abstract screening was undertaken for all citations. Citations missing an abstract during screening were retained for full-text screening to establish eligibility. Citations meeting eligibility criteria were included for full-text screening. Full-text screening followed the same strategy as the title and abstract screening to ensure interrater reliability.

Data extraction was performed by the same reviewers (SAL, PEA) for all included articles, independently and in duplicate. A third reviewer was available to resolve any disagreement between the two reviewers; however, all disagreements were resolved via consensus without involving a third reviewer.

\section{Quality appraisal}

The Mixed Methods Appraisal Tool (MMAT [25]) guided the quality appraisal for all qualitative, quantitative, and mixed methods studies. Quantitative and qualitative studies were each assessed by four criteria with overall scores varying from $0 \%$ (no criterion met) to $100 \%$ (all four criteria met). For mixed methods studies, three components were appraised: qualitative, quantitative, and mixed methods component, with the overall score determined by the lowest component score. In keeping with integrative review methods [24, 26], all records were retained in the analysis, regardless of score. For each article, two reviewers assessed methodological quality independently and discrepancies were resolved via consensus.

\section{Data analysis}

For each study, the steps of data reduction, data display, data comparison, and drawing conclusions and verifications were followed [24]. To ensure trustworthiness and rigor during data abstraction and synthesis, a table was developed to summarize the organizational contextual features. The abstracted information was compared, and patterns of findings were recorded as they emerged, followed by groupings of similar data and the identification of several key themes [24].

Analysis followed a qualitative descriptive approach, given that most of the study designs were qualitative and the results from quantitative studies could not be aggregated due to heterogeneity in study design, outcomes, and type of intervention [27]. Inductive content analysis was used to uncover themes related to organizational context $[28,29]$. This analytic approach involved reading and rereading the articles to uncover any salient codes and categories, subsequently collapsing them into themes (organizational contextual features) [27].

\section{Results}

Figure 1 depicts the search and screening phases as per Preferred Reporting Items for Systematic Reviews and Meta-Analyses (PRISMA) guidelines [30]. The search yielded 5152 citations. Following a review of titles and abstracts, 46 relevant articles were retrieved in full text and reviewed for eligibility. Of these, 10 were excluded because they did not explicitly explore and/or measure organizational context $(n=7)$, were part of quality improvement but did not include implementation of EBPs $(n=2)$, or were based on a system-level study $(n=1)$. Thirty-six peer-reviewed journal articles were included in the integrative review. The studies were methodologically diverse; 11 (30.6\%) were quantitative studies that explored organizational context as an outcome using cross-sectional surveys, 20 (55.6\%) were qualitative studies that described organizational context using themes derived from interviews and/or focus groups, and $5(13.9 \%)$ were mixed methods studies.

Table 1 Inclusion and exclusion criteria

\begin{tabular}{ll}
\hline Inclusion criteria & Exclusion criteria \\
\hline Articles were included if they: & Articles were excluded if they: \\
Published in a peer-reviewed journal & Were outside the healthcare domain \\
$\begin{array}{l}\text { Investigated contextual features at the organizational level as a primary or } \\
\text { secondary study objective }\end{array}$ & Did not investigate a KT initiative \\
& Were editorials, opinions, conceptual papers, discussions, or \\
Focused on implementation (including adoption, uptake, and research & textbooks \\
utilization) & Were reported in languages other than English \\
Empirical studies of all design types; \\
$\begin{array}{l}\text { Were published since } 2005, \text { to capture a wave of research on organizational } \\
\text { context over the past 12 years [78-80] }\end{array}$ \\
\hline
\end{tabular}




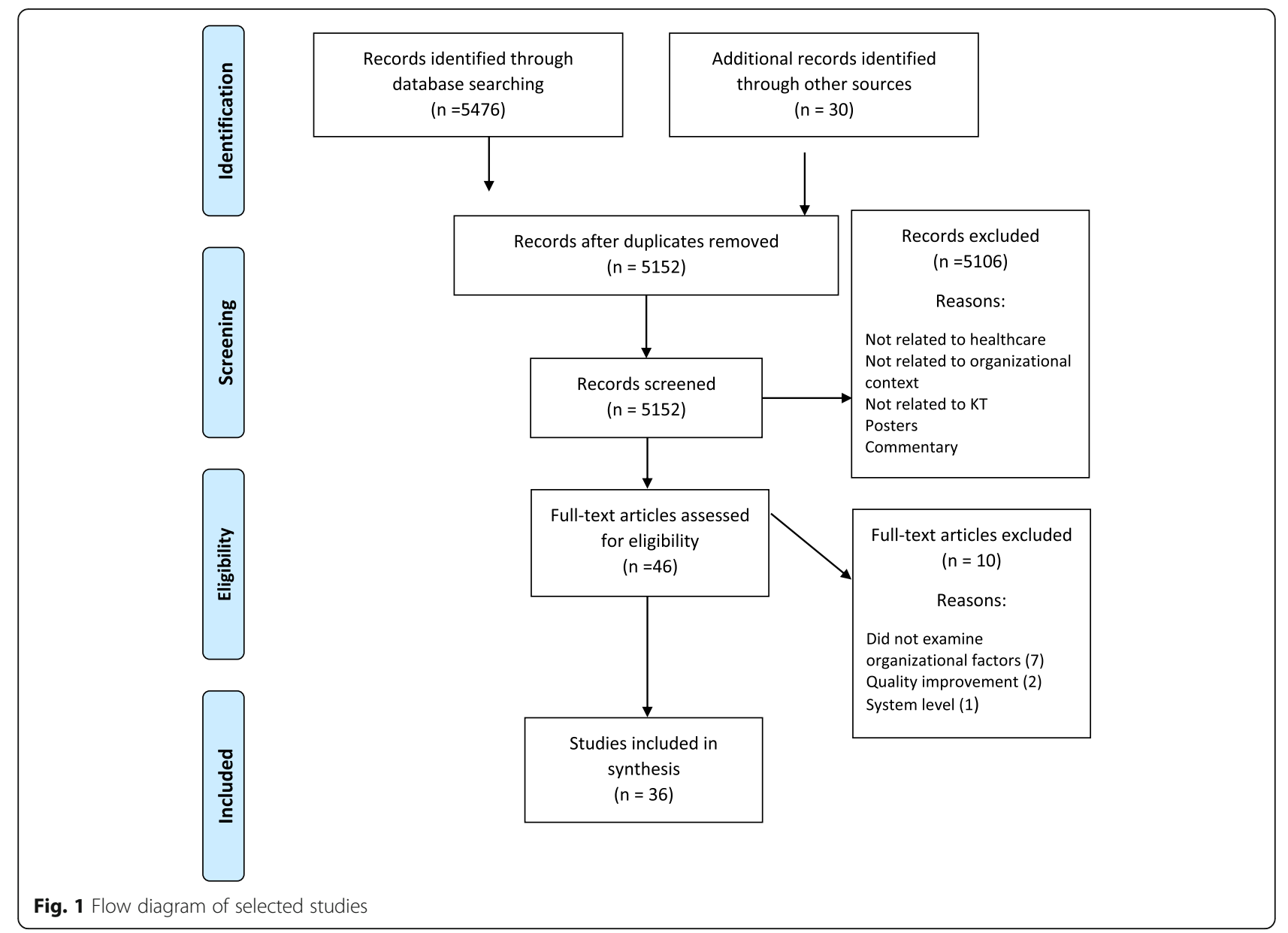

Even though implementation success was frequently mentioned in the included studies, none defined implementation success.

\section{Description of studies}

Table 2 presents the characteristics of the included studies, including study setting, study aim, sample, guiding framework (if applicable), study design and data collection methods, main findings, and MMAT quality score. The studies were published between 2007 and 2017 and were based in 11 countries. At least 8094 participants were included in this review. Study participants comprised a wide range of stakeholders including physicians, nurses, and allied health professionals working as coordinators, medical staff, and senior managers from many different healthcare settings. Two reported on the number of participating pediatric hospital units $(n=16)$ and medical centers $(n=12)$ instead of the number of individual participants. Twenty (56\%) of 36 studies used a theory, model, or framework to guide their data collection and/or analysis.

\section{Methodological quality}

The included studies were of moderate to high methodological quality (Table 1) based on the MMAT [25] appraisal. Of the 36 studies, 22 received a score of $75 \%$ (moderately high quality), 8 received 50\% (moderate quality), and 5 received 100\% (high quality).

\section{Organizational contextual features in empirical studies}

Six organizational contextual features included organizational culture; networks and communication; leadership; resources; evaluation, monitoring, and feedback; and champions. A series of sub-features included collaboration, teamwork, communication, financial resources, time, staffing and workload, and education and training. Table 3 illustrates the features and sub-features.

\section{Organizational culture}

Organizational culture was included as an organizational contextual feature in 22 of 36 (61\%) studies. Organizational openness to trialing new innovations and a learning culture were highly associated with implementation success [30-35]. Conversely, an absence of a learning culture can act as a major hindrance to 


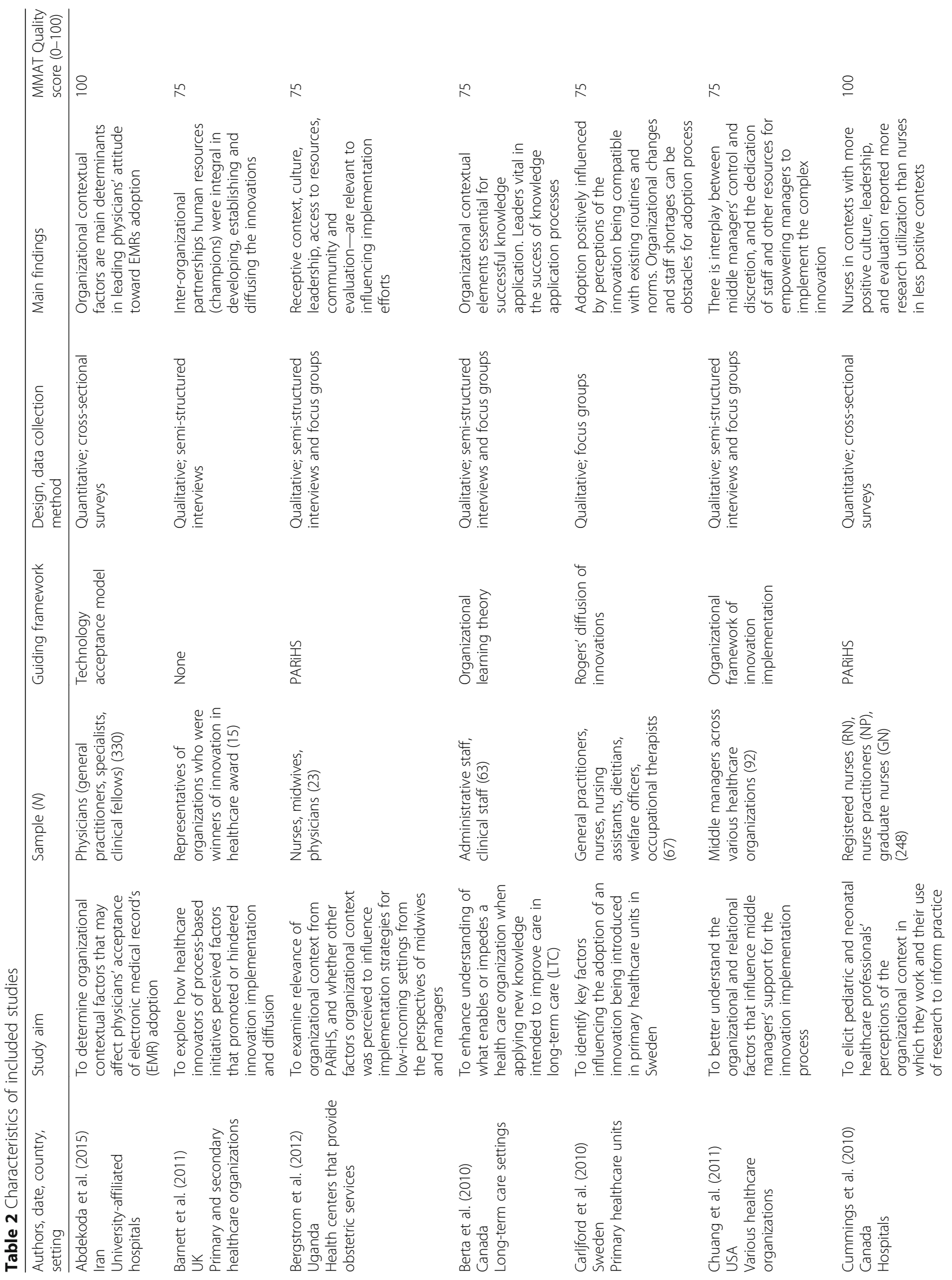




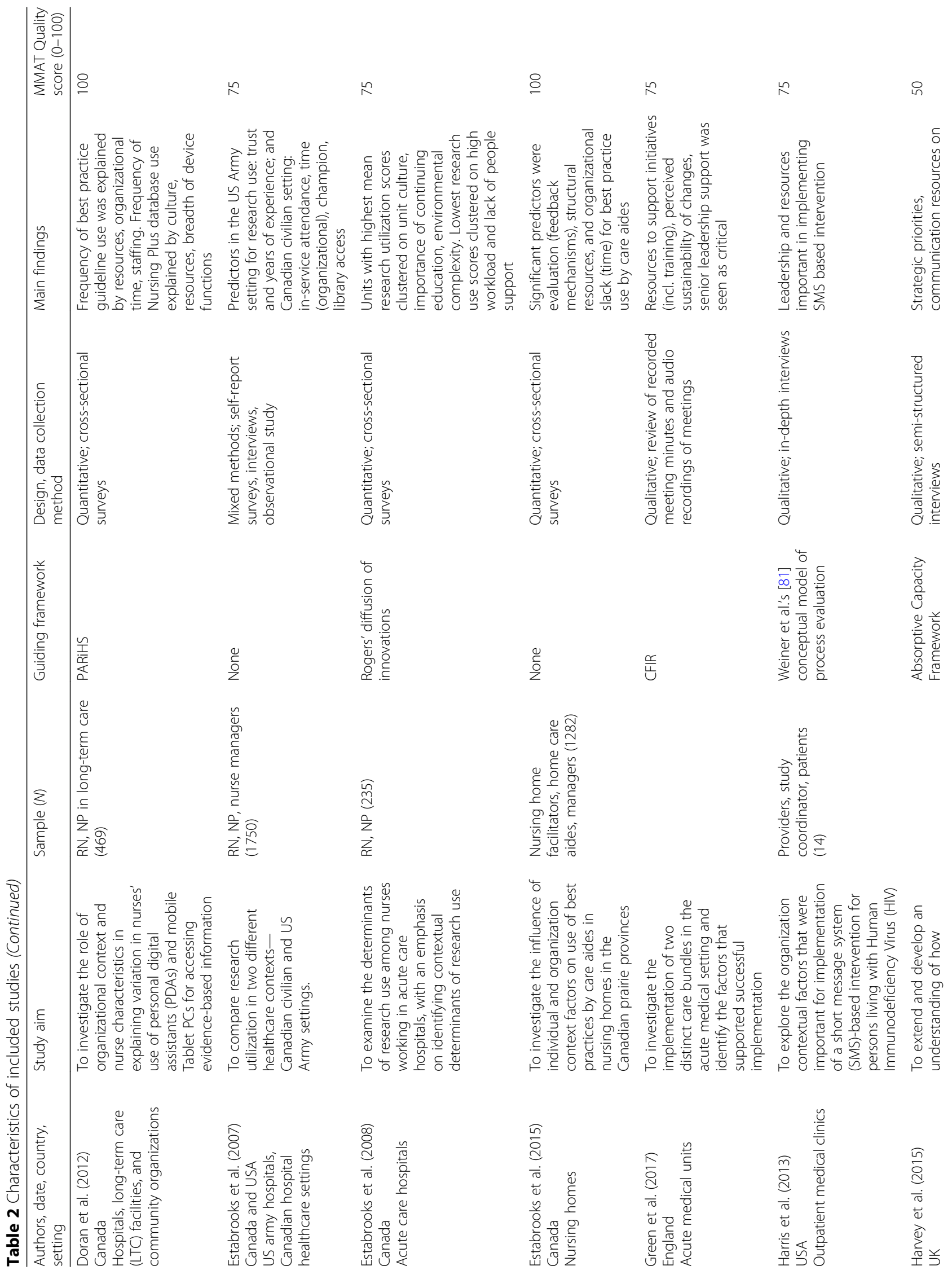




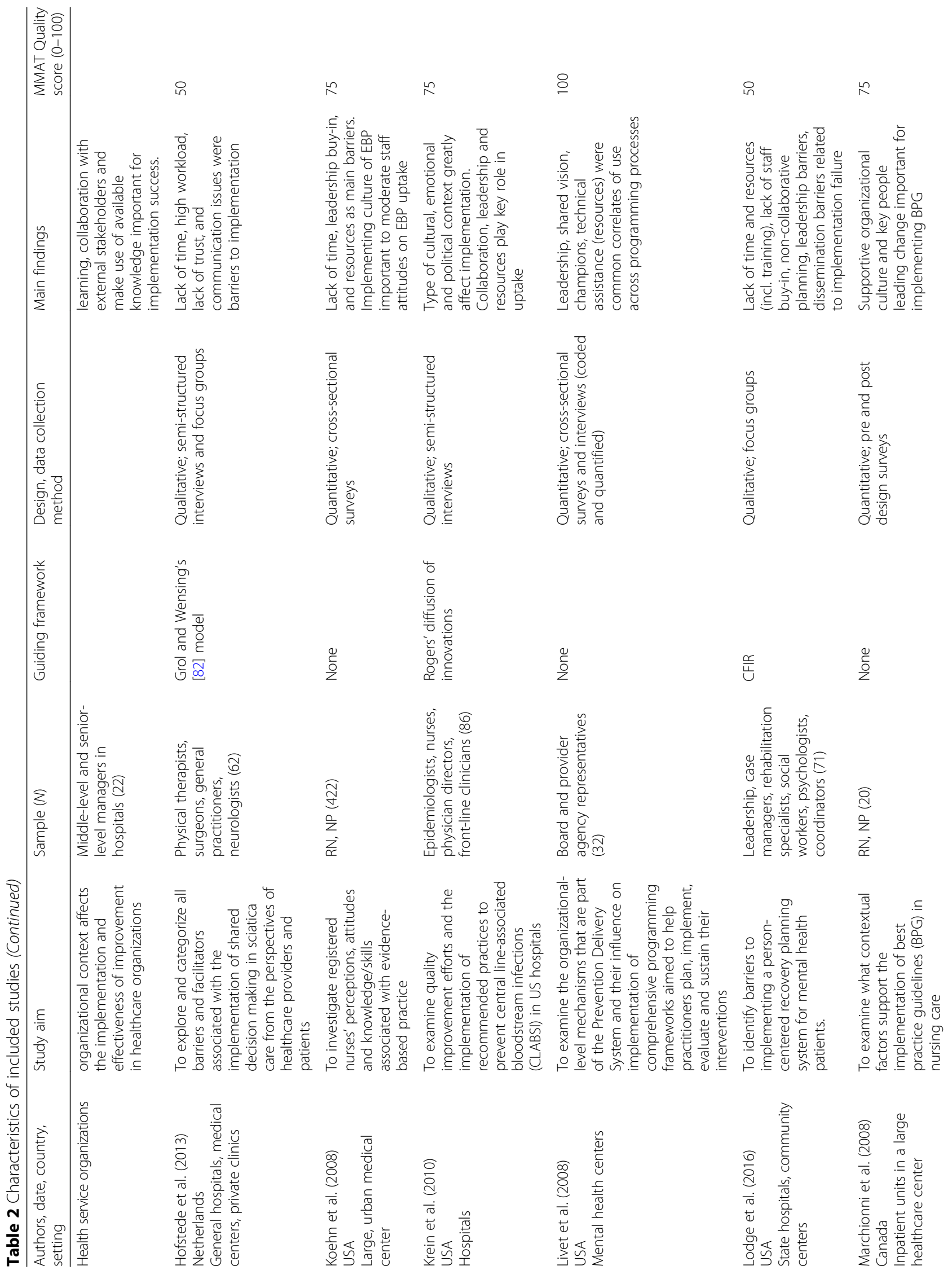




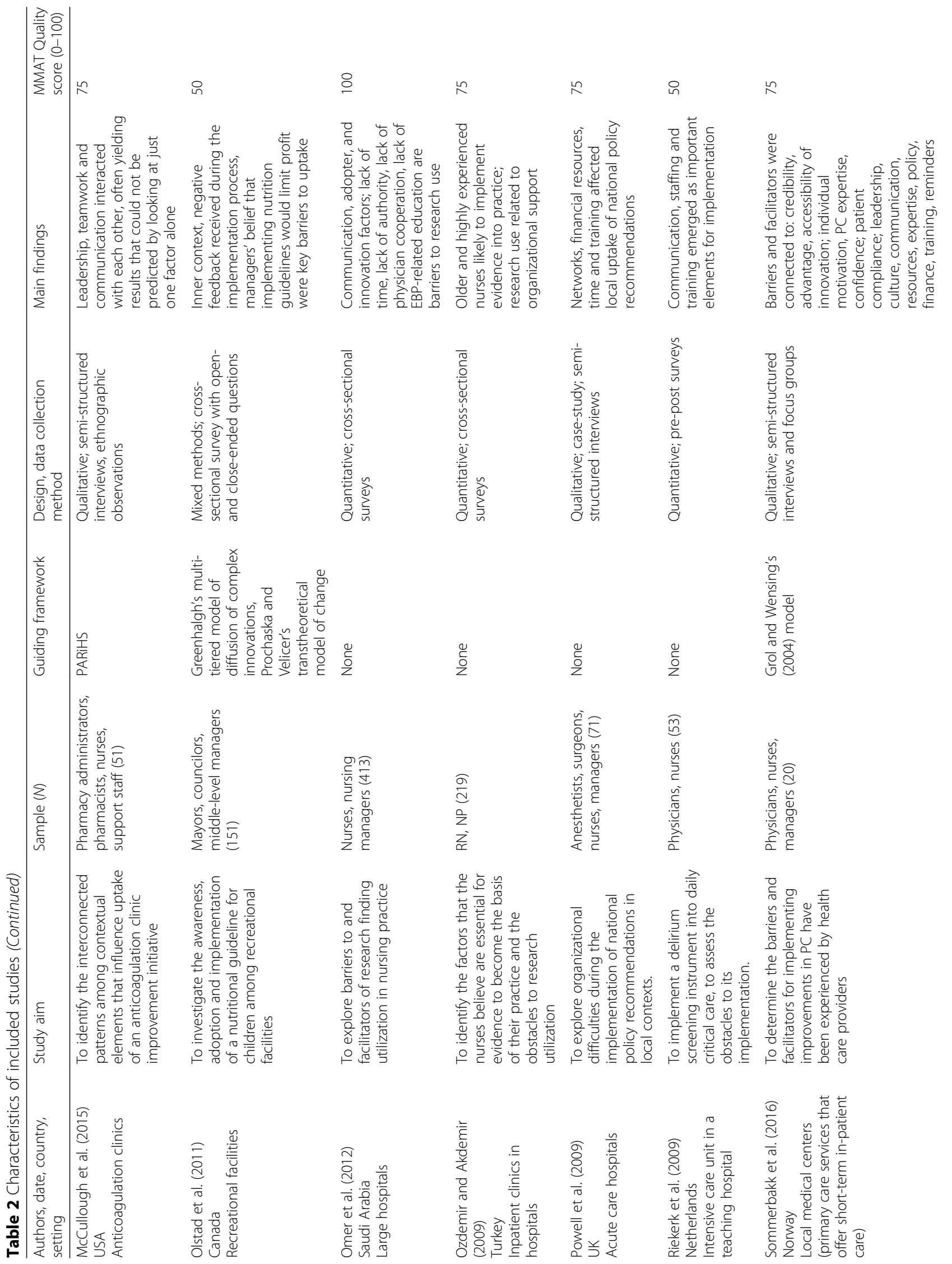




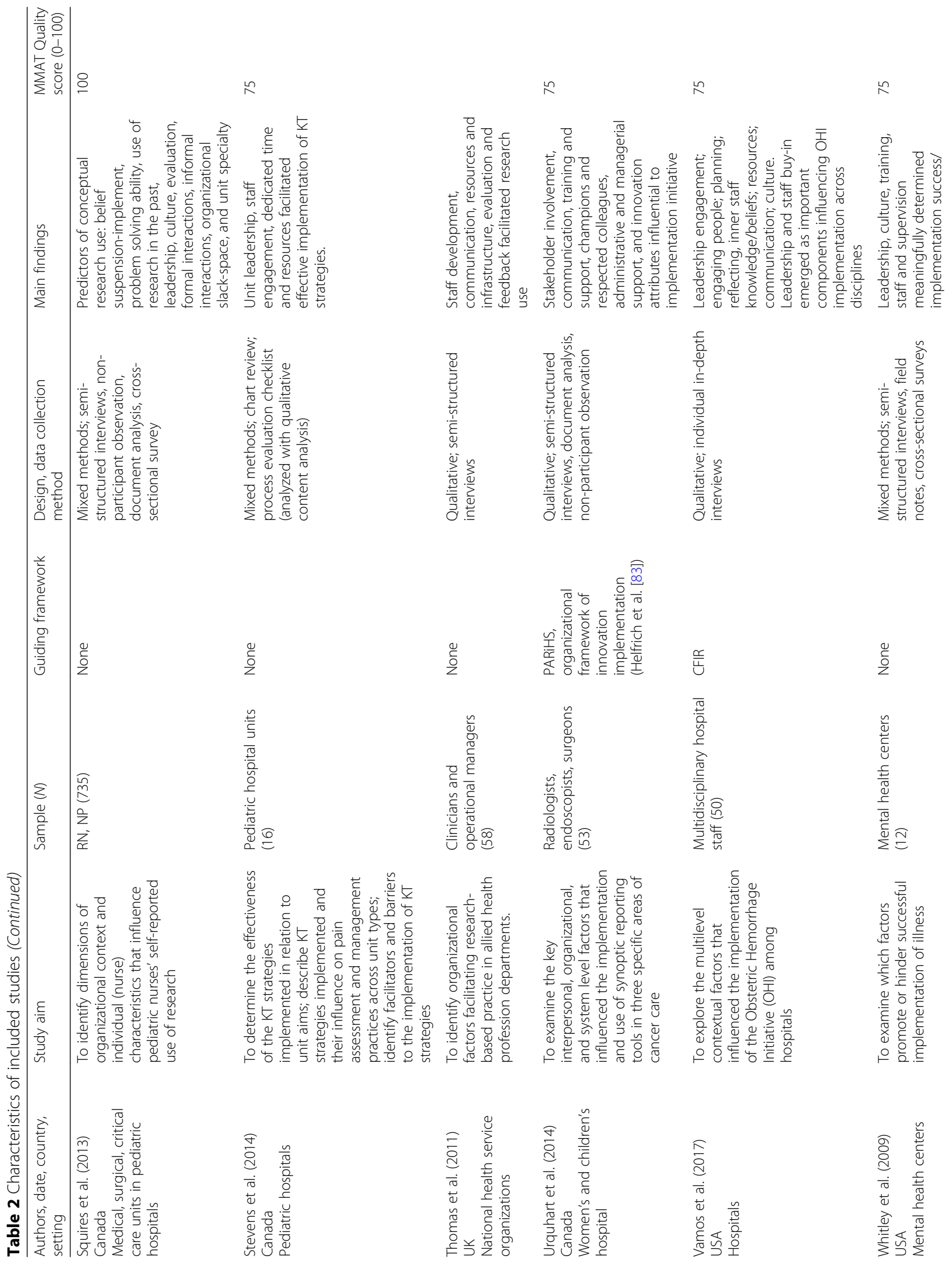




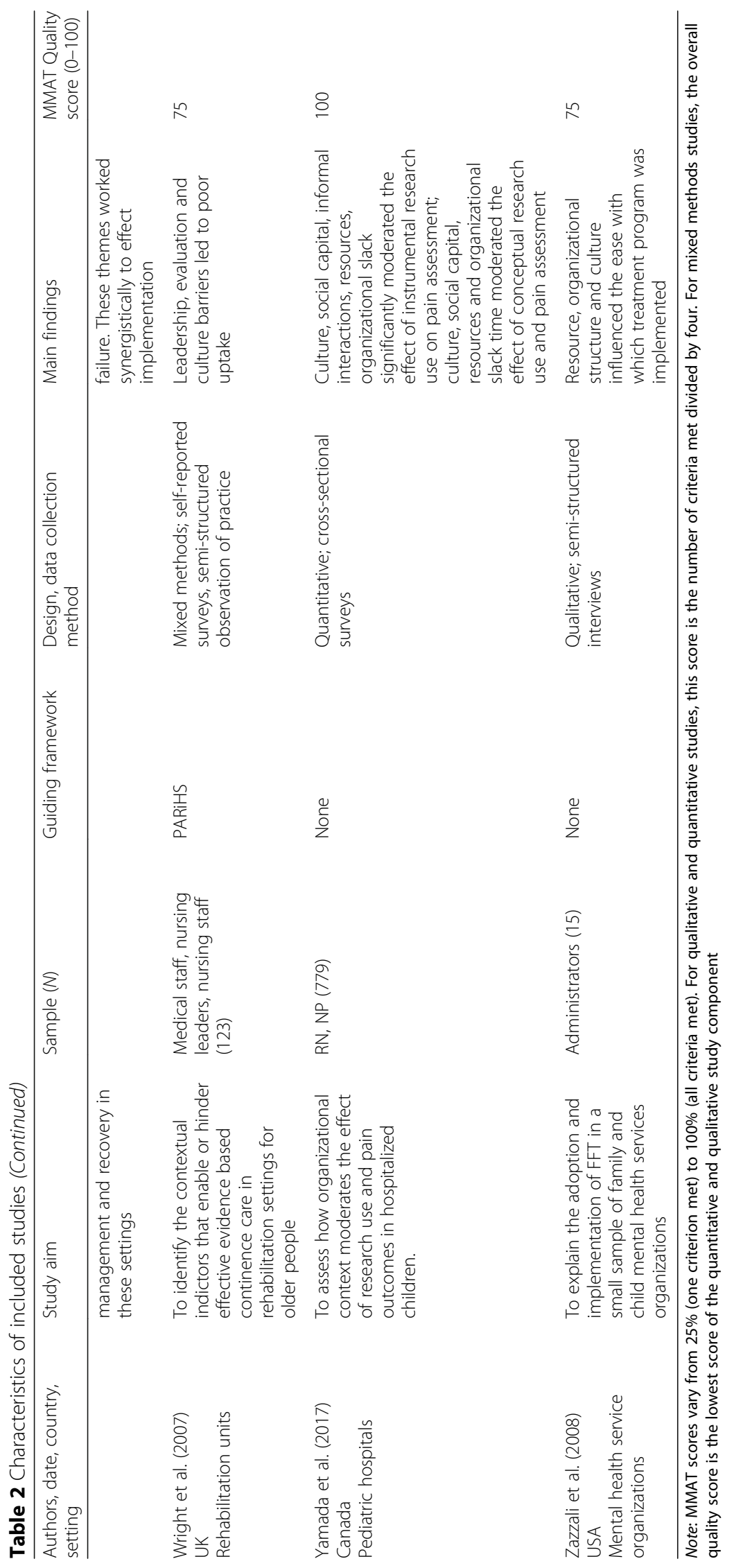


Table 3 Number of studies that reported on each feature, and their corresponding references

\begin{tabular}{lll}
\hline Features and sub-features & Number of studies out of 36 & Reference \\
\hline $\begin{array}{l}\text { Organizational culture } \\
\text { Networks and communication }\end{array}$ & 22 & {$[3,31-36,38-43,49,51-53,56,84,85] ;$} \\
$\begin{array}{l}\text { Leadership } \\
\text { Resources }\end{array}$ & 22 & {$[3,31,33,36-38,40,42,43,45-50,52-54,58,85,86] ;$} \\
$\quad$ Financial resources & 17 & {$[32-34,37-40,42-45,48-50,52,55,56,84,85] ;$} \\
$\quad$ Staffing and workload & 14 & {$[3,33,35-37,44,46,50,52,53,54-56,59,84,86] ;$} \\
$\quad$ Time & 13 & {$[32,35,36,38,41,43,47-50,57,84] ;$} \\
$\quad$ Education and training & 12 & {$[35,37,42,48,51,53,55,57-59,85,86] ;$} \\
Evaluation, monitoring, and feedback & 14 & {$[32,33,37-39,46,48,51-54,56,84] ;$} \\
Champion & 11 & {$[31,32,36,39,42-44,48,49,51,52,56,85] ;$} \\
\hline
\end{tabular}

successful implementation [36]. Organizational cultures comprised of staff who have too much autonomy (i.e., physicians experiencing a high level of autonomy when making decisions about how to treat patients) [37], are resistant to trial new innovations [38], or are unclear about organizational values and beliefs [39] can be barriers to successful implementation. Sites demonstrating high implementation fidelity were marked by a strong culture of innovation, accompanied by positive staff attitudes and behavior toward the new initiative [40]. In one study, unit culture (measured by work creativity, work efficacy, questioning behavior, co-worker support, and emphasis on continuing education) was a significant predictor of nurses' research use [41]. These results corroborated with other studies investigating organizational context and nurses' research utilization [32, 42]. Organizational culture significantly moderated the effect of nurses' instrumental (direct use of research knowledge) and conceptual research use (indirect use of research knowledge) on pediatric pain assessment in hospitals [43].

\section{Networks and communication}

An association of organizational networks and communication with implementation success was evident in 22 of 36 studies (61\%). Three sub-features were commonly associated with implementation outcomes.

\section{Collaborations}

Collaborative relationships that occur within and external to the organization were important for carrying out implementation plans. For instance, Barnett et al. highlighted two main purposes of interorganizational collaborations. First, materially based partnerships provided the organization with the resources required for the implementation and diffusion of new programs. Second, symbolically based interorganizational collaborations allowed organizations to gain local consensus to bolster the new programs with legitimacy, which in turn serves as an important social exchange that assisted with communicating the innovation's impact through gaining a broader consensus. Harvey et al. [39] described how close collaboration with an external implementation improvement team can support staff and leadership development geared toward implementing change.

\section{Teamwork}

Teamwork was characterized as good working relations, the ability to communicate clearly and effectively, and the capacity to solve problems together during EBP uptake [44-47]. Using an ethnographic case study design, McCullough et al. [47] observed that strong teamwork among staff, when combined with strong belief in evidence, led to high adoption of a dosing algorithm in anticoagulation clinics. However, when staff were dismissive of the evidence, strong teamwork served to reinforce resistance to implementation efforts. Teamwork was highly relevant in new programs that required participation from professionals in multidisciplinary teams. In a mental health organization where multidisciplinary staff (peer specialists, practitioners) were required to implement a new person-centered recovery program for their patients, poor multidisciplinary teamwork resulted in poor program uptake [38].

\section{Communication}

Communication greatly impacted the implementation of EBPs [37, 39, 44, 46, 48-50]. The establishment of systems and processes to more effectively manage information and communication about the change initiative influenced implementation success [39]. Communication between healthcare professionals in a Dutch intensive care unit (ICU) was an important barrier for a successful start of the implementation phase of a delirium scoring system [48]. Vamos et al. [50] and Stevens et al. [49] articulated various communication channels that facilitated implementation in hospital units, including active (scheduled meetings, debriefings, emails) and passive 
(flyers, announcements on bulletin boards, autogenerated reminders) communications.

\section{Leadership}

Leadership was reported in 20 of 36 (56\%) studies as an important feature for implementation effectiveness. Leaders were often seen as providers of new knowledge and as key influencers in new implementation initiatives [44]. Leaders who created environments with high staff morale allowed staff to perceive themselves as part of the implementation team. Transformational leadership often gave rise to clear roles and effective teamwork structures and cultivated a culture of learning [44]. Leaders ensured changes were sustained, without which staff were reported to "fall back into the old ways of doing things" [37]. Senior leaders were important for ensuring that new processes were integrated as "business as usual" [33]. Senior leaders were also essential for overall hospital staff involvement and buy-in [33, 37, 50]. The initial decision to begin an implementation effort within the hospital and the subsequent ongoing changes during the implementation process required the engagement of leadership at different levels and from multiple stakeholders across hospital departments [50]. The willingness of middle managers to partake in the implementation process was often contingent upon the support expressed by senior leaders [31]. The absence of senior leader support or tension between middle managers and their direct supervisors meant middle managers were significantly more reluctant to participate. Leaders work to optimize implementation success by expressing enthusiasm for the change; being present, supportive, and attentive to the implementation process; and demonstrating willingness to ask for feedback from staff regarding the change. Leadership that is lacking in authority and unsupportive of change, or that neglects to hold staff accountable for the change, presents barriers to implementation $[38,51]$. Staff feel unmotivated to change when leaders were too controlling or unresponsive to requests for more training by staff who were required to implement the practice change [40].

\section{Resources}

This feature is divided into four sub-features that are interrelated and appear to work synergistically to hinder or promote the implementation process.

\section{Financial resources}

Financial resources were highly important to the implementation process in 17 of 36 studies (47\%). Lack of sufficient dedicated funding among acute pain specialized teams meant they struggled to provide adequate service across different departments and sites, leaving no funding reserves for promoting and integrating new innovations. Time that could have been allocated to activities like training and educating staff on the EBP was instead used for seeking funds for other initiatives [51]. Urquhart et al. [52] reported that limited financial resources, including financially dependent resources (e.g., acquiring personnel), were a key constraining feature in implementing a new synoptic reporting tool in different surgery departments. Securing adequate funding to train and educate staff on the new initiative [37-39, 53], allocating human resources to make the change [29, 30, 52], providing monitoring and feedback to ensure fidelity at the change sites [45], and ensuring a smooth transition for the implementation (i.e., new equipment or services to accommodate for the change) $[54,55]$ were crucial to optimize implementation effectiveness.

\section{Staffing and workload}

Thirteen of 36 studies (36\%) reported on the effects of staffing and workload on the implementation process. Staff experiencing heavy workloads or insufficient staffing on normal routine activities were less likely to carry out change [34, 39, 42, 48, 56, 57]. Assigning dedicated staff to perform the change was associated with successful implementation. Dedicating staff time to implementation activities facilitated effective implementation for pediatric pain management [49] and for obstetrics hemorrhage cases in hospitals [50].

Chuang et al. [31] described how insufficient staffing can be a major implementation challenge for middle managers. Those who could manage staff with little effect on normal working environments were significantly more likely to support the innovation. Middle managers play a key role in facilitating implementation, and their decisions about which staff should undergo training were a key determinant for implementation success [52]. High staff turnover is problematic for implementation, creating a never-ending cycle of training seminars and educational sessions that consume a significant amount of time and resources $[38,53]$.

\section{Time}

Thirteen of 36 studies (36\%) identified time constraints as a barrier to implementing EBPs. Time constraint was conceptualized at three levels. At the staff level, insufficient time due to other more urgent, competing demands often hindered the full implementation of EBPs $[31,58,59]$. At the innovation level, staff who perceived the EBP as more time-consuming than usual practice were more reluctant to adopt the EBP [57, 59]. Insufficient time for staff training, planning, and staff rescheduling (to implement the EBP) were barriers at the logistics level [36, 38, 49]. Conversely, having adequate time for these activities was positively associated with research use in practice $[43,54,60]$. 


\section{Education and training}

Education and training contributed to the effectiveness of EBP implementation in 12 of 36 (33\%) studies. Lack of training and development for the EBP among staff and local champions were key barriers to implementation success [38, 39, 51, 53]. Unclear or insufficient educational materials and reminders, inconsistent use of educational materials, and not having enough staff to participate in educational outreach influenced the implementation success of evidence-based pain research in hospital units to varying extents [49]. Staff were more likely to participate in educational sessions and training initiatives if these were offered several times and if leadership mandated the training [53]. Whitley et al. [40] found that high-quality training of a new mental health illness management program by competent and respected trainers was a key factor in high-fidelity sites. Training also promoted interdisciplinary collaboration, since the continuous training sessions provided opportunities for communication and teamwork [52].

\section{Evaluation, monitoring, and feedback}

This feature was important for successful implementation in 14 of 36 studies (39\%). Appropriate feedback mechanisms benefited EBP implementation by preserving engagement among staff who implemented the change. Active and engaged leaders who sought feedback about the change and who provided feedback to staff were associated with higher rates of implementation success $[33,34,53]$. Soliciting early feedback from middle managers can help assuage their concerns about the change initiative, and ongoing staff communication and monitoring increased the likelihood of EBP sustainability over time [31]. Three studies reported that evaluation and feedback were important predictors of research use among nurses [32, 54, 60]. Evaluation moderated the effect of nurses' use of research for pain management [43]. Audit and feedback were effective for improving nursing practice in pain management and assessment for children [49].

\section{Champions}

Presence of champions was important for implementation success in 11 of 36 studies (31\%). Champion was the strongest and most consistent feature related to the use of a new systematic framework for prevention delivery services (including the use of implementation guidelines) [45]. Having a champion to advocate for the "new way of doing things" led to more complete and refined use of these guidelines. Supporting champions can be difficult in contexts that lack engaged leadership or dedicated resources to encourage and monitor adherence. Identified champions who rise to the challenge may succumb to feelings of frustrations when the organization does not support change. Key attributes of successful champions included the following: (a) being an expert on the EBP, (b) being available for troubleshooting and for training other staff "on the floor," and (c) providing a sense of familiarity among colleagues and belief in the champion's expertise. In one study, the management team chose staff members who were initially unsupportive of the implementation project and motivated them to take an active role in the project, which prevented them from thwarting the implementation progress [53].

\section{Interrelationships between organizational contextual features}

Potential interrelationships between features were identified in 12 of 36 studies. Figure 2 illustrates the direction of influence between each feature. Leadership influenced all other features of this review: (a) the use and selection of champions [33, 37, 40, 50], (b) the allocation of resources (funds and additional staffing) to accommodate for the implementation [36-38, 52, 53], (c) the facilitation or hindrance to the monitoring and feedback mechanisms during the implementation process $[52,53]$, and (d) organizational culture [34, 39, 50, 52].

Networks and communication worked synergistically with other features to promote or hinder the implementation process. For instance, an organizational culture of innovation can be cultivated by ongoing and explicit communication of new innovations [44]. Leaders can help champions communicate observable impacts of the new initiative to other staff, as observed in a study investigating the adoption of clinical practice guidelines in long-term care homes [37]. Similarly, communicating with middle managers and senior leadership to gain buyin for an EBP was a significant contributor to implementation success $[31,39]$.

Organizational culture interacted with other organizational contextual features. Bergström et al. [44] found that the organizational culture had set the stage for supportive, inter-professional teamwork and was more important than training staff about implementing the EBP. Sommerbakk et al. [53] demonstrated that a culture characterized by trust and open communication was a facilitator for EBP uptake. Chuang et al. [31] reported that a culture of learning builds teamwork and contributes positively to the performance of the hospital unit that is implementing change. On the contrary, lack of support from colleagues was a barrier to constructing a change culture [53]. Strong leadership, coupled with a culture of learning or openness to innovation, was important to successful implementation [31, 32, 34, 35, 37, 40, 41, 43, 52].

Leadership, culture, resources, and networks and communication contributed to implementation success in at least $50 \%$ of the 36 selected studies; among these, 12 studies (33\%) identified at least one feature or a sub- 


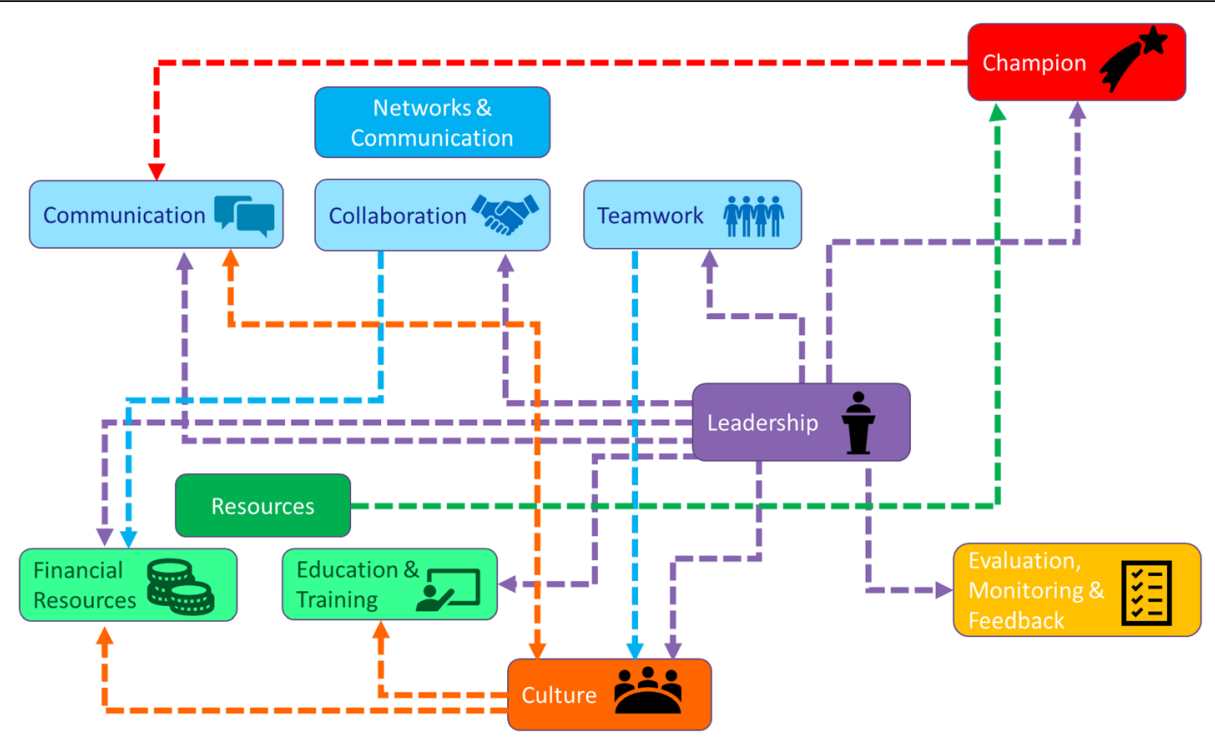

Fig. 2 Illustration of the relationships between organizational contextual features and sub-features based on analysis of the results of selected studies. Arrows depict the potential direction of the relationship (e.g., leadership influences evaluation and feedback). The color of each dotted line corresponds to the feature that may exert influence on the other connecting feature. Please refer to the main text for a description of these potential interrelationships between the features and sub-features

feature that influenced or worked synergistically to either act as an enabler or a constraint to the implementation process.

\section{Discussion}

This integrative review identified six organizational contextual features that are important to EBP implementation across healthcare settings. Implementation process is influenced by the organizational culture, leadership, communication and networks, resources, champions, and evaluation, monitoring, and feedback activities within healthcare organizations. Organizational contextual features did not influence implementation efforts independently from other features. Rather, features were interrelated and often influenced each other in complex, dynamic ways to effect change. This finding is congruent with the CFIR, which asserts that the inner setting constructs (discrete theoretical concepts) are interrelated and influence implementation [15]. Given that the six organizational contextual features corresponded to the CFIR inner setting (constructs: culture, networks and communication, resources, leadership engagement) and process (constructs: reflecting and evaluating, champions) domains, the CFIR may serve as an appropriate framework for assessing or improving organizational context in a wide range of healthcare settings. Notably, the CFIR identified 39 constructs, which go well beyond the six features that were identified from this review. Identifying the most commonly reported features that influence the implementation provides preliminary evidence that these may be the most important for optimizing implementation effectiveness.

Kirk et al. [61] and May et al.'s [62] systematic review of studies that used the CFIR as a guiding framework found variation in the use of CFIR constructs, but these studies rarely justified their choice. Justifying which CFIR constructs to investigate can help ensure the consistency of implementation studies and allows researchers to compare these studies over time and across different settings $[15,61,62]$. Findings of this review can provide preliminary guidance for selecting which contextual features to modify during implementation planning. Nonetheless, readers should keep in mind how these contextual features were identified in the included studies. Over half $(56 \%)$ of the included studies were guided by frameworks and measures or applied existing theoretical perspectives. Researchers of these included studies may be sensitized to specific contextual features or constructs, which may have precluded examination of other features beyond those illustrated in the guiding theory, framework, or model. Many included studies did not define organizational context, and those that were guided by frameworks, theories, or models conceptualized organizational context differently.

Without a single operational definition, studies claiming to investigate organizational context may be examining different constructs. Researchers suggested that incomplete definitions of context, combined with inconsistencies in definitions, have led to conceptual overlap and confusion in the specification of context $[63,64]$. Measuring and assessing a core set of contextual 
features across healthcare settings can allow for a synthesis of findings across studies to detect trends that consistently influence implementation outcomes. By operationally defining organizational context, implementation researchers can advance the existing limited evidence base on understanding how contextual features can affect implementation and under which conditions. Findings of this review can provide some indication on how implementation health researchers are operationally defining organizational context.

The six contextual features combine to promote or hinder implementation depending on their presence or absence. Organizations that comprise low-fidelity implementation sites usually involve the absence or malfunction of one or more of these features. Capitalizing on these features most likely supports implementation activities. The finding that organizational contextual features synergistically influenced implementation efforts supports that context is not just a physical setting or a backdrop for implementation; organizational contextual features interact, impact, modify, promote, or hinder the EBP and its implementation efforts. Furthermore, the EBP, target users, implementation process, and inner and outer contexts are intertwined, constantly interacting with and influencing each other [15]. The interrelationships between organizational contextual features support Aarons et al.'s [65] postulation that context should not be viewed as a fixed, organizational structure or institutional entity but as an unstable, unfolding process.

Organizational culture was most commonly reported to affect EBP implementation. However, extant literature suggests very limited, if any, interventions to improve organizational culture in healthcare settings [66]. Culture exerts influence on available resources such as funding and educational support, and can be modified by the type of leadership (i.e., transformational versus authoritarian), level of communication (i.e., low versus high), and quality of teamwork within the organization. One study examining mental health clinician attitudes on EBPs found that more engaged organizational cultures and implementation climates, characterized by higher levels of educational support, coupled with more interactive implementation leadership were associated with more positive attitudes toward EBPs [67]. The researchers examined implementationspecific organizational constructs (e.g., implementation climate, implementation leadership) that are more proximal to implementation. The relationship between general organizational context (as reviewed in this paper) and implementation-specific organizational context has yet to be fully established. Future implementation strategies should address features that are associated with both general and implementation-specific organizational contexts to explore their potential roles as mediators and moderators of implementation effectiveness [68].
Leadership influences all other features, suggestings that it may be a priority feature in implementation efforts. Empirical evidence supports the critical importance of leaders in the implementation process [69, 70] and considers leadership as essential for creating an organizational context conducive to change [71, 72]. There is a need to better understand how leadership interacts with other key features associated with implementation success so that resources can be meaningfully directed to shape the contextual features that have high impact on implementation outcomes.

\section{Limitations}

This review was limited to published journal articles in English; the results may have limited transferability to non-English-speaking nations that have very different healthcare systems. This review was also limited to studies that investigated organizational contextual features during the implementation, adoption, and uptake phases of EBPs; these studies provided little understanding of how organizational contextual features impact the sustainability of EBPs. The search strategy of this review used the term "context" in the organization to identify empirical studies that investigated organizational context. However, it is likely that other researchers who examined the same organizational contextual features identified in this review may not use the term "context" in their report. As such, these studies could not be retrieved. For example, Williams et al. [73] reported an increase in EBP uptake through improved organizational culture among mental health clinicians in 14 children's mental health agencies. This study was not captured in this review but proves to be highly relevant to inform implementation researchers about the value of organizational culture change on implementation effectiveness. Guerrero et al. [74] observed that the leader's openness to and expectations about implementing EBPs were strongly associated with the implementation of a contingency management strategy in substance abuse treatment programs. Therefore, readers should approach the review findings with caution, bearing in mind the limitations of the search strategy in this review.

Several limitations at the level of individual studies warrant discussion. This review identified potential interrelationships between the organizational contextual features but did not explore the nature of these relationships, with one exception [46]. Study findings were reported very briefly in the "Results" sections, which precluded reviewers from drawing further conclusions about these interrelationships. The extent to which these features may be more effective for implementation if considered in concert or individually remains an empirical question that needs further exploration. The organization contextual features identified as consistently influential to implementation efforts were 
contingent upon the study authors' decisions as to which features belonged at the organizational level. It is possible that other less frequently explored contextual features can also influence implementation outcomes.

Although the term "implementation success" frequently appeared in the "Results" and "Discussion" sections of the included studies, this term was not defined. Implementation success can be measured or conceptualized differently in different healthcare settings. Implementation studies should describe how "implementation success" is conceptualized or operationalized in the implementation project, or report on any pre-determined targets that represent implementation effectiveness. Most of the included studies used qualitative approaches to identify, describe, or explain the organizational contextual features that emerged from this review; however, it was unclear whether the conceptual or operational definitions for each of these features (e.g., culture, leadership) were consistent across the included studies. Defining each feature being investigated will enhance the clarity and consistency of the feature and facilitate external validity.

Even though 27 out of 36 included studies were rated as moderately high to high quality according to the MMAT, the included studies did not follow any standard methods of reporting, which is consistent with existing literature that articulated the low reporting standards of implementation studies [74, 75]. Implementation researchers should consider using the Standards for Reporting Implementation Studies (StaRI) [76] to ensure transparent and accurate reporting of implementation studies. StaRI requires researchers to provide an extensive description of context, which will help readers assess the external validity of the reported study, and decide how the implementation context in the study compares to their own setting. A rich description of the study's implementation context is crucial to readers who are considering whether the implementation strategy can be directly adopted or will need modifications [77].

\section{Conclusions}

This integrative review provides an overview of how implementation researchers operationalized organizational context in healthcare settings, and describes the potential interrelationships among the six most commonly reported organizational contextual features that influence EBP implementation. Shared commonality in how we define, assess, and measure organizational context can add to the generalizability of future studies. A core set of organizational contextual features influencing the implementation of EBPs exist across a wide range of healthcare settings. These organizational contextual features were consistent with the constructs illustrated in CFIR [15], supporting its use as a guiding framework for exploring the relationship between organizational contextual features and implementation. Future research needs to confirm this finding and examine the interrelationships between different contextual features which, by working together, can act as enablers in one implementation setting but barriers in others. Accounting for interconnections among organization contextual features at each KT phase may enable implementation researchers to more fully describe the determinants of successful implementation in clinical practice. Developing a conducive organizational context, specifically with strong leadership capacity, can be an essential precursor to facilitate the implementation of EBPs in a wide range of healthcare settings.

\section{Additional file}

Additional file 1: Literature search strategy (MEDLINE). (DOCX 13 kb)

\section{Abbreviations}

CFIR: Consolidated Framework for Implementation Research; CINAHL: Cumulative Index to Nursing and Allied Health Literature; EBP: Evidence-based practice; EMBASE: Excerpta Medica Database; HCP: Healthcare professional; ICU: Intensive care unit; KT: Knowledge translation; MEDLINE: Medical Literature Analysis and Retrieval System Online; MMAT: Mixed Methods Appraisal Tool; PARiHS: Promoting Action Research in Health Services; PRISMA: Preferred Reporting Items for Systematic Reviews and Meta-Analyses; PsycINFO: American Psychological Association's (APA) resource for abstracts of scholarly journal articles, book chapters, books, and dissertations; StaRI: Standards for Reporting Implementation Studies

\section{Acknowledgements}

The authors of this review are very grateful to Dr. Paul Elias Alexander (PEA) for his participation in the screening of eligible studies, data extraction, and quality assessment components of the review. We would also like to acknowledge the financial support of the Canadian Institutes of Health Research (CIHR) Foundation Grant (\#148452) and Pain In Child Health (PICH) CIHR Strategic Training in Health Research Grant (\#53885).

\section{Funding}

Funding was provided by the Canadian Institutes of Health Research (CIHR) Foundation Grant (\#148452) and Pain In Child Health (PICH) CIHR Strategic Training in Health Research Grant (\#53885).

\section{Availability of data materials}

The datasets used and/or analyzed during the current study are available from the corresponding author on a reasonable request.

\section{Authors' contributions \\ SAL developed the idea of the review, conducted the literature search, analyzed and interpreted all the data, drafted the manuscript, and served as the primary role of editing and revising of subsequent iterations of the manuscript. $\sqcup$ overlooked the literature search process, provided input in the conduct of the search strategy, revised the manuscript critically for important intellectual content, and provided the final approval of the version to be published. MB provided input in the conduct of the search strategy, revised the manuscript critically for important intellectual content, and provided the final approval of the version to be published. BJS overlooked the entire research project, revised the manuscript critically for important intellectual content, and provided the final approval of the version to be published. All authors read and approved the final manuscript.}

Ethics approval and consent to participate

Because this manuscript reports findings of an integrative review of empirical studies, ethics approval and consent to participate is not needed. 


\section{Consent for publication}

Because the manuscript does not contain any individual person's data in any form, consent for publication is not needed.

\section{Competing interests}

The authors declare that they have no competing interests.

\section{Publisher's Note}

Springer Nature remains neutral with regard to jurisdictional claims in published maps and institutional affiliations.

\section{Author details}

${ }^{1}$ Lawrence S. Bloomberg Faculty of Nursing, University of Toronto, Toronto, ON, Canada. ${ }^{2}$ St Michael's Hospital Volunteer Association Chair in Nursing Research, Li Ka Shing Knowledge Institute, Toronto, ON, Canada. ${ }^{3}$ Lawrence S. Bloomberg Faculty of Nursing and Institute of Health, Policy Management and Evaluation, University of Toronto, Toronto, Canada. ${ }^{4}$ Child Health Evaluative Sciences, Research Institute, Peter Gilgan Centre for Research and Learning, The Hospital for Sick Children, Toronto, ON, Canada. ${ }^{5}$ Department of Psychiatry, University of Toronto, Toronto, Canada. ${ }^{6}$ The Dalla Lana School of Public Health, University of Toronto, Toronto, Canada. ${ }^{7}$ Faculties of Medicine and Dentistry, University of Toronto Centre for the Study of Pain, University of Toronto, Toronto, Canada.

\section{Received: 19 December 2017 Accepted: 18 April 2018} Published online: 05 May 2018

\section{References}

1. Research America. U.S. investments in medical and health research and development: 2013-2015. 2015. https://www.researchamerica.org/sites/ default/files/2016US_Invest_R\%26D_report.pdf. Accessed 17 June 2017.

2. Canadian Institutes of Health Research. 2016-17 Report on Plans and Priorities. 2016. http://cihr-irsc.gc.ca/e/49658.html. Accessed 17 June 2017.

3. Barnett J, Vasileiou K, Djemil F, Brooks L, Young T. Understanding innovators' experiences of barriers and facilitators in implementation and diffusion of healthcare service innovations: a qualitative study. BMC Health Serv Res. 2011;11(1):342.

4. Sackett DL, Rosenberg WM, Gray JM, Haynes RB, Richardson WS. Evidence based medicine: what it is and what it isn't. Br Med J. 1996:312:71-2.

5. Glasgow RE, Vinson C, Chambers D, Khoury MJ, Kaplan RM, Hunter C. National Institutes of Health approaches to dissemination and implementation science: current and future directions. Am J Public Health. 2012;102(7):1274-81

6. Morris ZS, Wooding S, Grant J. The answer is 17 years, what is the question: understanding time lags in translational research. J R Soc Med. 2011:104(12):510-20

7. Grimshaw JM, Eccles MP, Lavis JN, Hill SJ, Squires JE. Knowledge translation of research findings. Implement Sci. 2012;7(1):50.

8. Dobbins M, Robeson P, Ciliska D, Hanna S, Cameron R, O'Mara L, DeCorby K, Mercer S. A description of a knowledge broker role implemented as part of a randomized controlled trial evaluating three knowledge translation strategies. Implement Sci. 2009;4(1):23.

9. Pfadenhauer LM, Gerhardus A, Mozygemba K, Lysdahl KB, Booth A, Hofmann B, Wahlster P, Polus S, Burns J, Brereton L, Rehfuess E. Making sense of complexity in context and implementation: the context and implementation of complex interventions (CICI) framework. Implement Sci. 2017;12(1):21.

10. Cummings GG, Estabrooks CA, Midodzi WK, Wallin L, Hayduk L. Influence of organizational characteristics and context on research utilization. Nurs Res. 2007:56(4):S24-39.

11. Eccles MP, Armstrong D, Baker R, Cleary K, Davies H, Davies S, Glasziou P, llott I, Kinmonth AL, Leng G, Logan S. An implementation research agenda. Implement Sci. 2009;4(1):18.

12. Dee JR, Heineman WA. Understanding the organizational context of academic program development. New Directions for Institutional Research. 2016;2015(168):9-35.

13. Porter LW, McLaughlin GB. Leadership and the organizational context: like the weather? Leadersh Q. 2006;17(6):559-76.

14. Rusu G, Avasilcăi S, Huțu CA. Organizational context factors influencing employee performance appraisal: a research framework. Procedia-Social and Behavioral Sciences. 2016;221:57-65.
15. McNulty T, Ferlie E. Reengineering health care: the complexities of organizational transformation. OUP Oxford; 2002. http://www. oxfordscholarship.com/view/10.1093/acprof:oso/9780199269075.001.0001/ acprof-9780199269075.

16. Warde A. Cultural consumption, classification and power. London: Routledge; 2013.

17. Rogers EM. Diffusion of innovations. New York: Free Press; 2003. p. 551.

18. Damschroder LJ, Aron DC, Keith RE, Kirsh SR, Alexander JA, Lowery JC Fostering implementation of health services research findings into practice: a consolidated framework for advancing implementation science. Implement Sci. 2009;4(1):50.

19. Aarons GA, Hurlburt M, Horwitz SM. Advancing a conceptual model of evidence-based practice implementation in public service sectors. Adm Policy Ment Health Ment Health Serv Res. 2011;38(1):4-23.

20. Harvey G, Kitson A. PARIHS revisited: from heuristic to integrated framework for the successful implementation of knowledge into practice. Implement Sci. 2016:11(1):33.

21. Francis JJ, O'Connor D, Curran J. Theories of behaviour change synthesised into a set of theoretical groupings: introducing a thematic series on the theoretical domains framework. Implement Sci. 2012;7(1):35.

22. Squires JE, Graham ID, Hutchinson AM, Michie S, Francis JJ, Sales A, et al. Identifying the domains of context important to implementation science: a study protocol. Implement Sci. 2015;10:135. https://doi.org/10.1186/s13012-015-0325-y.

23. Broome ME. Integrative literature reviews for the development of concepts. Concept development in nursing: foundations, techniques and applications Philadelphia: WB Saunders Company; 2000. p. 231-50.

24. Whittemore R, Knafl K. The integrative review: updated methodology. J Adv Nurs. 2005;52(5):546-53.

25. Pluye P, Robert E, Cargo M, Bartlett G, O'cathain A, Griffiths F, Boardman F, Gagnon MP, Rousseau MC. Proposal: a mixed methods appraisal tool for systematic mixed studies reviews. Montréal: McGill University; 2011. p. 1-8.

26. Ganong LH. Integrative reviews of nursing research. Research in Nursing \& Health. 1987;10(1):1-1.

27. Sandelowski M. Focus on research methods-whatever happened to qualitative description? Research in Nursing and Nealth. 2000;23(4):334-40.

28. Altheide DL. Reflections: ethnographic content analysis. Qual Sociol. 1987; 10(1):65-77.

29. Morgan DL. Qualitative content analysis: a guide to paths not taken. Qual Health Res. 1993;3(1):112-21.

30. Moher D, Liberati A, Tetzlaff J, Altman DG, Prisma Group. Preferred Reporting Items for Systematic Reviews and Meta-Analyses: the PRISMA statement. PLoS Med. 2009 Jul 21;6(7):e1000097.

31. Chuang E, Jason K, Morgan JC. Implementing complex innovations: factors influencing middle manager support. Health Care Manag Rev. 2011;36(4):369-79.

32. Cummings GG, Hutchinson AM, Scott SD, Norton PG, Estabrooks CA. The relationship between characteristics of context and research utilization in a pediatric setting. BMC Health Serv Res. 2010;10(1):168.

33. Green SA, Bell D, Mays N. Identification of factors that support successful implementation of care bundles in the acute medical setting: a qualitative study. BMC Health Serv Res. 2017;17(1):120.

34. Krein SL, Damschroder $\sqcup$, Kowalski CP, Forman J, Hofer TP, Saint S. The influence of organizational context on quality improvement and patient safety efforts in infection prevention: a multi-center qualitative study. Soc Sci Med. 2010;71(9):1692-701.

35. Marchionni $\mathrm{C}$, Ritchie J. Organizational factors that support the implementation of a nursing best practice guideline. J Nurs Manag. 2008;16(3):266-74.

36. Zazzali JL, Sherbourne C, Hoagwood KE, Greene D, Bigley MF, Sexton TL. The adoption and implementation of an evidence based practice in child and family mental health services organizations: a pilot study of functional family therapy in New York state. Adm Policy Ment Health Ment Health Serv Res. 2008;35(1-2):38-49.

37. Berta W, Teare GF, Gilbart E, Ginsburg LS, Lemieux-Charles L, Davis D, Rappolt $\mathrm{S}$. The contingencies of organizational learning in long-term care: factors that affect innovation adoption. Health Care Manag Rev. 2005;30(4):282-92.

38. Lodge AC, Kaufman L, Manser SS. Barriers to implementing person-centered recovery planning in public mental health organizations in Texas: results from nine focus groups. Adm Policy Ment Health Ment Health Serv Res. 2017:44(3):413-29.

39. Harvey G, Jas P, Walshe K. Analysing organisational context: case studies on the contribution of absorptive capacity theory to understanding inter-organisational variation in performance improvement. BMJ Qual Saf. 2014;0:1-8. 
40. Whitley R, Gingerich S, Lutz WJ, Mueser KT. Implementing the illness management and recovery program in community mental health settings: facilitators and barriers. Psychiatr Serv. 2009;60(2):202-9.

41. Squires JE, Estabrooks CA, Scott SD, Cummings GG, Hayduk L, Kang SH, Stevens B. The influence of organizational context on the use of research by nurses in Canadian pediatric hospitals. BMC Health Serv Res. 2013;13(1):351.

42. Estabrooks CA, Scott S, Squires JE, Stevens B, O'Brien-Pallas L, WattWatson J, Profetto-McGrath J, McGilton K, Golden-Biddle K, Lander J, Donner $G$. Patterns of research utilization on patient care units. Implement Sci. 2008;3(1):31.

43. Yamada J, Squires JE, Estabrooks CA, Victor C, Stevens B. The role of organizational context in moderating the effect of research use on pain outcomes in hospitalized children: a cross sectional study. BMC Health Serv Res. 2017;17(1):68.

44. Bergström A, Peterson S, Namusoko S, Waiswa P, Wallin L. Knowledge translation in Uganda: a qualitative study of Ugandan midwives' and managers' perceived relevance of the sub-elements of the context cornerstone in the PARIHS framework. Implement Sci. 2012;7(1):117.

45. Livet $M$, Courser $M$, Wandersman $A$. The prevention delivery system: organizational context and use of comprehensive programming frameworks. Am J Community Psychol. 2008;41(3-4):361-78.

46. McCullough MB, Chou AF, Solomon JL, Petrakis BA, Kim B, Park AM, Benedict AJ Hamilton $A B$, Rose AJ. The interplay of contextual elements in implementation: an ethnographic case study. BMC Health Serv Res. 2015;15(1):62.

47. Powell AE, Davies HT, Bannister J, Macrae WA. Understanding the challenges of service change-learning from acute pain services in the UK. J R Soc Med. 2009;102(2):62-8.

48. Riekerk B, Pen EJ, Hofhuis JG, Rommes JH, Schultz MJ, Spronk PE. Limitations and practicalities of CAM-ICU implementation, a delirium scoring system, in a Dutch intensive care unit. Intensive and Critical Care Nursing. 2009;25(5):242-9.

49. Stevens BJ, Yamada J, Promislow S, Stinson J, Harrison D, Victor JC Implementation of multidimensional knowledge translation strategies to improve procedural pain in hospitalized children. Implement Sci. 2014;9(1):120.

50. Vamos CA, Thompson EL, Cantor A, Detman L, Bronson E, Phelps A, Louis JM, Gregg AR, Curran JS, Sappenfield W. Contextual factors influencing the implementation of the obstetrics hemorrhage initiative in Florida. J Perinatol. 2017:37(2):150-6.

51. Omer T. Research utilization in a multicultural nursing setting in Saudi Arabia: barriers and facilitators. J Nurs Res. 2012;20(1):66-73.

52. Urquhart R, Porter GA, Sargeant J, Jackson L, Grunfeld E. Multi-level factors influence the implementation and use of complex innovations in cancer care: a multiple case study of synoptic reporting. Implement Sci. 2014;9(1):121.

53. Sommerbakk R, Haugen DF, Tjora A, Kaasa S, Hjermstad MJ. Barriers to and facilitators for implementing quality improvements in palliative care-results from a qualitative interview study in Norway. BMC Palliative Care. 2016;15(1):61.

54. Doran D, Haynes BR, Estabrooks CA, Kushniruk A, Dubrowski A, Bajnok I, Hall LM, Li M, Carryer J, Jedras D, Bai YQ. The role of organizational context and individual nurse characteristics in explaining variation in use of information technologies in evidence based practice. Implement Sci. 2012;7(1):122.

55. Thomas L, Roddam H, Baker P, French B, Burton C. Indicators facilitating research-based practice in allied health profession departments. International Journal of Therapy \& Rehabilitation. 2011;1:18(2).

56. Harris JL, Furberg R, Martin N, Kuhns L, Lewis MA, Coomes C, Williams P, Uhrig JD. Implementing an SMS-based intervention for persons living with human immunodeficiency virus. Journal of Public Health Management and Practice. 2013;19(2):E9-16.

57. Koehn ML, Lehman K. Nurses' perceptions of evidence-based nursing practice. J Adv Nurs. 2008;62(2):209-15.

58. Carlfjord S, Lindberg M, Bendtsen P, Nilsen $P$, Andersson A. Key factors influencing adoption of an innovation in primary health care: a qualitative study based on implementation theory. BMC Fam Pract. 2010;11(1):60

59. Hofstede SN, Marang-van de Mheen PJ, Wentink MM, Stiggelbout AM Vleggeert-Lankamp CL, Vlieland TP, van Bodegom-Vos L. Barriers and facilitators to implement shared decision making in multidisciplinary sciatica care: a qualitative study. Implement Sci. 2013;8(1):95.

60. Estabrooks CA, Squires JE, Hayduk L, Morgan D, Cummings GG, Ginsburg L, Stewart N, McGilton K, Kang SH, Norton PG. The influence of organizational context on best practice use by care aides in residential long-term care settings. J Am Med Dir Assoc. 2015;16(6):537-e1.
61. Kirk MA, Kelley C, Yankey N, Birken SA, Abadie B, Damschroder L. A systematic review of the use of the consolidated framework for implementation research. Implement Sci. 2016;11(1):72.

62. May CR, Johnson M, Finch T. Implementation, context and complexity. Implementation Science. 2016;11(1):141.

63. Gershon RR, Stone PW, Bakken S, Larson E. Measurement of organizational culture and climate in healthcare. The Journal of Nursing Administration. 2004;34:33-40.

64. Sleutel MR. Climate, culture, context, or work environment? Organizational factors that influence nursing practice. J Nurs Adm. 2000;30:53-8.

65. Aarons GA, Horowitz JD, Dlugosz LR, Ehrhart MG. The role of organizational processes in dissemination and implementation research. In: Brownson RC, Colditz GA, Proctor EK. (eds.). Dissemination and implementation research in health: translating science to practice. Oxford, England: Oxford University Press; 2012. p.128-153.

66. Parmelli E, Flodgren G, Beyer F, Baillie N, Schaafsma ME, Eccles MP. The effectiveness of strategies to change organisational culture to improve healthcare performance: a systematic review. Implement Sci. 2011;6(1):33.

67. Powell BJ, Mandell DS, Hadley TR, Rubin RM, Evans AC, Hurford MO, Beidas RS. Are general and strategic measures of organizational context and leadership associated with knowledge and attitudes toward evidence-based practices in public behavioral health settings? A cross-sectional observational study. Implement Sci. 2017;12(1):64.

68. Williams NJ. Multilevel mechanisms of implementation strategies in mental health: integrating theory, research, and practice. Adm Policy Ment Health Ment Health Serv Res. 2016;43(5):783-98.

69. Gifford W, Davies B, Edwards N, Griffin P, Lybanon V. Managerial leadership for nurses' use of research evidence: an integrative review of the literature. Worldviews Evid-Based Nurs. 2007:4(3):126-45

70. Aarons GA, Sommerfeld DH. Leadership, innovation climate, and attitudes toward evidence-based practice during a statewide implementation. Journal of the American Academy of Child \& Adolescent Psychiatry. 2012;51(4):423-31.

71. Battilana J, Gilmartin M, Sengul M, Pache AC, Alexander JA. Leadership competencies for implementing planned organizational change. Leadersh Q. 2010;21(3):422-38.

72. Aarons GA, Ehrhart MG, Farahnak LR, Hurlburt MS. Leadership and organizational change for implementation (LOCI): a randomized mixed method pilot study of a leadership and organization development intervention for evidence-based practice implementation. Implement Sci. 2015;10(1):11.

73. Williams NJ, Glisson C, Hemmelgarn A, Green P. Mechanisms of change in the ARC organizational strategy: increasing mental health clinicians' EBP adoption through improved organizational culture and capacity. Adm Policy Ment Health Ment Health Serv Res. 2017;44(2):269-83.

74. Guerrero EG, He A, Kim A, Aarons GA. Organizational implementation of evidence-based substance abuse treatment in racial and ethnic minority communities. Adm Policy Ment Health Ment Health Serv Res. 2014:41(6):737-49.

75. Pinnock H, Epiphaniou E, Pearce G, Parke H, Greenhalgh T, Sheikh A, Griffiths CJ, Taylor SJ. Implementing supported self-management for asthma: a systematic review and suggested hierarchy of evidence of implementation studies. BMC Med. 2015;13(1):127.

76. Rycroft-Malone J, Burton CR. Is it time for standards for reporting on research about implementation? Worldviews Evid-Based Nurs. 2011;8(4):189-90

77. Pinnock H, Barwick M, Carpenter CR, Eldridge S, Grandes G, Griffiths CJ, Rycroft-Malone J, Meissner P, Murray E, Patel A, Sheikh A. Standards for reporting implementation studies (StaRI) statement. BMJ. 2017;356:i6795.

78. Graham ID, Tetroe J. Some theoretical underpinnings of knowledge translation. Acad Emerg Med. 2007;14(11):936-41.

79. Jacobson N, Butterill D, Goering P. Development of a framework for knowledge translation: understanding user context. Journal of Health Services Research \& Policy. 2003;8(2):94-9.

80. Greenhalgh T, Robert G, Macfarlane F, Bate P, Kyriakidou O. Diffusion of innovations in service organizations: systematic review and recommendations. The Milbank Quarterly. 2004;82(4):581-629.

81. Weiner BJ, Megan AL, Laura AL. "Using organization theory to understand the determinants of effective implementation of worksite health promotion programs." Health Educ Res. 2008;24(2):292-305.

82. Grol R, Michel W. "What drives change? Barriers to and incentives for achieving evidence-based practice." Med J Aust. 2004;180(6 Suppl):S57.

83. Helfrich $C D$, et al. "Determinants of implementation effectiveness: adapting a framework for complex innovations." Med Care Res Rev. 2007;64(3):279-303. 
84. Wright J, McCormack B, Coffey A, McCarthy G. Evaluating the context within which continence care is provided in rehabilitation units for older people. Int J Older People Nursing. 2007;2(1):9-19.

85. Estabrooks CA, Midodzi WK, Cummings GG, Wallin L. Predicting research use in nursing organizations: a multilevel analysis. Nurs Res. 2007;56(4):S7-23.

86. Özdemir L, Akdemir N. Turkish nurses' utilization of research evidence in clinical practice and influencing factors. Int Nurs Rev. 2009;56(3):319-25.

- fast, convenient online submission

- thorough peer review by experienced researchers in your field

- rapid publication on acceptance

- support for research data, including large and complex data types

- gold Open Access which fosters wider collaboration and increased citations

- maximum visibility for your research: over $100 \mathrm{M}$ website views per year 